\title{
EL ALCANCE DE LAS NOCIONES NORMATIVAS DENTRO DE LA CIENCIA MÉDICA
}

\author{
Guillermo J. MAÑÓN GARIBAY
}

Resumen:

Salud/enfermedad son tanto conceptos centrales de la ciencia médica como de importancia para la sociedad en general. El presente artículo trata de la interacción de ambas concepciones con respecto a su influencia normativa.

Abstract:

Health and illness are two central concepts in the medical field and are important to society in general as well. This article studies the interaction between the two conceptions in relation to its normative importance. 
SumARIO: I. Introducción. II. Peculiaridades de la ciencia médica. III. La relación conocer/obrar en la medicina. IV. La relación entre conocer y obrar en la medicina moderna. V. Nueva lectura de la ciencia médica desde los conceptos normativos salud/enfermedad. VI. La teoría de la medicina frente a la apertura interpretativa de salud y enfermedad. VII. Relación entre teoría y ética médica con base en los conceptos salud/enfermedad.

\section{INTRODUCCIÓN}

El presente estudio se propone mostrar el vínculo que existe entre saber médico y práctica médica, así como sobre el sentido y función de los conceptos salud/enfermedad. Esto a su vez será un aporte a la compresión y relevancia de una conexión entre, por un lado, teoría de la medicina y, por otro, ética e historia de la medicina, porque se considera que sólo una teoría de la medicina informada de la historia y de la ética de la medicina está en condiciones de dar cuenta de todos los problemas que surgen de la práctica médica.

Comenzaré con el planteamiento más elemental:

1. ¿Qué significa hacer una teoría de la medicina?

2. ¿En qué reside su tarea y su finalidad?

Lo primero que salta a la vista es la ambigüedad respecto del campo de adscripción de la teoría de la medicina; porque ésta puede representar los intereses del historiador, asi como los de la filosofía práctica y también de la ciencia médica, pero incluso del médico mismo. De esta forma, se puede entender por teoría de la medicina al menos cuatro cosas distintas:

1) Reflexión ético-jurídica sobre la práctica médica, como una práctica justificada en un saber del cuerpo y dirigida por los conceptos normativos salud/enfermedad. 
2) Reflexión filosófica sobre la capacidad de la medicina para explicar el organismo humano y, tal vez, curarlo.

3) Reflexión histórica sobre el quehacer médico y las distintas teorías sobre el cuerpo dentro de las distintas culturas.

4) Por último, reflexión sobre una determinada teoría dentro de la medicina, con la que se intenta resolver, científica y clínicamente, problemas concretos de la práctica médica.

En este trabajo se entenderá la teoría de la medicina de acuerdo con sus tres primeras acepciones. De tal forma que teoría de la medicina equivale a filosofía de la ciencia médica en cuanto ésta explica, cura y norma las funciones del cuerpo. Lo que implica, en primera instancia, que el poder normativo y curativo de la medicina depende de su poder explicativo.

\section{PECUliaridades DE LA CIENCIA MÉdicA}

La medicina se distingue de las ciencias naturales en que la reflexión y análisis de la teoría médica versa sobre la práctica médica. La medicina, al contrario de la astronomía, no se puede disociar de su aplicación a casos particulares. Por esto mismo, la medicina se construye a lo largo de su práctica o ejercicio. La consecuencia de esta peculiaridad es que la teoría de la medicina no pude partir del estudio de conceptos y condiciones teóricas, porque la praxis médica no se orienta a partir de presupuestos y axiomas teóricos, sino por los resultados alcanzados en la práctica misma.

Teniendo esto en cuenta, puede preguntarse cuáles son los temas principales para la teoría de la medicina. En primera instancia, puede decirse que un aspecto principal es la relación entre el conocer y el quehacer médicos. La importancia de este aspecto se hace patente cuando se atiende a otra peculiaridad más, a saber: la práctica médica ope- 
ra con los conceptos salud/enfermedad. Lo que significa que la relación entre conocer y obrar se da dentro de un contexto normativo. ${ }^{1}$ Entonces, además del aspecto práctico, también el aspecto normativo tiene importancia teórica.

Este último aspecto distingue mayormente la teoría médica de cualquier otro estudio científico sobre el organismo humano, porque impide que la práctica médica se reduzca a simple biología humana.

Si la teoría de la medicina estudia la práctica médica en su relación con el conocer, y referido al contexto normativo salud/enfermedad, entonces la teoría médica pretende resolver dos problemas:

1. Hasta qué punto el saber sobre el organismo humano justifica la práctica médica (relación conocer/obrar).

2. Hasta qué punto el poder del saber médico reglamenta su obrar, en el marco de los conceptos normativos salud/enfermedad.

\section{LA RELACIÓN CONOCER/OBRAR EN LA MEDICINA}

A partir del siglo XVI y hasta entrado el siglo XIX se opera un cambio en la medicina consistente en convertir la experimentación en un elemento esencial de su desarrollo. ${ }^{2}$ Este cambio fue determinante para entender la relación entre conocimiento y quehacer médico, porque a lo largo de ese tiempo se fue considerando progresivamente a la experimentación como condición sine qua non del avance científico. Pero también, por otro lado, la práctica médica debió ser controlada por el saber científico a manera de garante del trabajo médico.

El profesor Ruy Pérez Tamayo informa lo siguiente al respecto:

1 Canguilhem, G., Lo normal y lo patológico, México, Siglo XXI, 1971, segunda parte, p. 83.

2 Babini, J., Historia de la medicina, Barcelona, Gedisa, 2000, cap. 12, pp. 61 y ss.; y Porter, R., Breve historia de la medicina. De la antigüedad hasta nuestros dias, Madrid, Taurus, 2004. 
Al terminar la Edad Media los enfermos tenían tres fuentes posibles de ayuda para el diagnóstico y tratamiento de sus males: 1) el médico, educado en una universidad de orientación galénica o arabista, que se limitaba a hacer diagnósticos y pronósticos y a recetar pócimas y menjunjes como la teriaca, y que no ejercía la cirugía porque para ingresar a la universidad (París, Montpellier) había tenido que jurar que no lo haría; 2) el cirujano-barbero, que no había asistido a una universidad sino que se había educado como aprendiz de otro cirujano barbero más experimentado; 3) el curandero, charlatán o mago, un embaucador itinerante, que viajaba de pueblo en pueblo vendiendo sus ungüentos y sus talismanes, sacando dientes ocasionalmente, hasta haciendo flebotomías y cirugía menor, casi siempre con resultados desastrosos. ${ }^{3}$

Este panorama nada halagüeño manifiesta la necesidad de unir conocimiento con práctica médica. De esta manera, la experiencia fue tanto el detonador del conocimiento médico, así como también el punto de verificación de ese conocimiento. Es manifiesto desde la primera fase del cambio hacia la medicina científica, que el conocimiento fue asumido como el factor "normativo" de la práctica médica: lo debido en la práctica médica correspondía con lo sabido por el médico. Si a esta consideración se agrega la concepción de "conocimiento científico" de principios del modernismo, inspirada puntualmente en los criterios mecanicistas ${ }^{4}$ impuestos por Rene Descartes, ${ }^{5}$ entonces se entiende el efecto restringido de la medicina para el hombre y sociedad, expuestos por el profesor Ruy Pérez Tamayo.

Gracias a la revolución cartesiana se formaron nuevas escuelas de medicina como la iatromecánica, la iatroquímica, el animismo, el vitalismo, el solidismo, el brownismo, el

3 Pérez Tamayo, R., De la magia primitiva a la medicina moderna, México, Fondo de Cultura Económica, Ciencia para todos, n. 154, 2003, p. 87.

4 Véase Porte, R., Breve historia de la medicina. De la antigüedad hasta nuestros dias, cit., nota 2, pp. 113 y ss.

5 Williams, B., Descartes. The Project of Pure Enquiry, Nueva York, Penguin Books, 1978, pp. 261 y ss. 
mesmerismo, etcétera. Nuevas concepciones de medicina científica que disputarían a la teoría de los humores ${ }^{6}$ de Claudio Galeno su predominio sostenido durante 1500 años. El ejemplo más conspicuo es la iatromecánica, y sobre ella nos dice lo siguiente el doctor Pérez Tamayo: "La iatromecánica es la doctrina que compara al cuerpo humano con una máquina artificial y pretende explicar su funcionamiento sobre bases puramente físicas". ${ }^{7}$

Esta visión condicionó la práctica médica al criterio de ciencia obtenido de las matemáticas y la física. Lo que significó que cada caso clínico fuera revisado individualmente, bajo una observación guiada por criterios científicos "seguros" y sin ningún efecto aparente para la autonomía del ser pensante y su entorno. ${ }^{8}$

\section{LA RELACIÓN ENTRE CONOCER Y OBRAR EN LA MEDICINA MODERNA}

Actualmente continua siendo la investigación científica - en el sentido de ciencia experimental- el instrumento principal para la elaboración de conocimiento en la medicina. Desde hace tiempo sirve el conocimiento biomédico, obtenido con modernos métodos experimentales y tecnológicos, para controlar la calidad y seguridad de la práctica médica ex post, y también para proporcionar criterios con los cuales identificar y analizar los problemas de la práctica

6 Claudio Galeno (130-200 d. C.) adoptó y elaboró la teoría hipocrática de la enfermedad como un desequilibrio de los humores, resultado del exceso o deficiencia de uno o más de ellos. Los humores eran cuatro: cólera (o bilis amarilla), sangre, flema y bilis negra. A partir de ellos se crearon también teorías sobre el temperamento de los hombres: flemático, sanguíneo, colérico y melancólico. Véase Babini, J., Historia de la medicina, Gedisa, Barcelona, 2000, pp. 29 y ss.

7 Pérez Tamayo, op. cit., nota 3, p. 117.

8 Esto se entiende frente a la concepción desarrollada por R. Descartes sobre el hombre como ente compuesto de dos substancias distintas e independientes, una pensante y otra extensa. Véase Descartes, R., Meditaciones metafísicas. Sobre todo la Meditación sexta: "De la existencia de las cosas materiales y de la de la distinción real entre el alma y el cuerpo del hombre”, Gredos, Madrid, 1999, p. 230. 
médica. ${ }^{9}$ Esta última es precisamente una de las funciones esenciales del conocimiento científico: determinar, hasta cierto punto a priori, el tipo de casos clínicos que pueden ser aceptados por el médico. Sin embargo, lo dicho hasta ahora no deja ver lo compleja que es la relación entre conocimiento y praxis médica.

En la situación actual de la ciencia, impulsada cada vez más por la tecnología, ${ }^{10}$ la biomedicina elabora un conocimiento que no cesa de aumentar y hacerse más complejo $^{11}$. En ese crecimiento toman parte muchas disciplinas altamente especializadas de las ciencias naturales que no están forzosamente vinculadas a la investigación médica o clínica. Ejemplos de esto son la genética molecular o la bioinformática, porque, sin tener una relación directa con la medicina, transforman el quehacer médico resolviendo problemas mediante nuevas aplicaciones tecnológicas. Esto no sólo renueva sino además agudiza el problema de la relación entre conocimiento y práctica médica: investigaciones realizadas desde planteamientos hechos por la biología, química, física, informática, etcétera, son de interés médico aunque hayan surgidos fuera de la investigación médica. ${ }^{12}$ Esto crea una avalancha de información difícil de considerar y aplicar en la práctica médica. Por eso, lo importante para el presente trabajo es saber cuáles son las consecuencias de la afluencia masiva de información relevante para la práctica médica.

La primera de ellas es que el conocimiento científico, relevante para la toma de decisiones en la práctica médica, es tan extenso en cada especialidad médica que ni siquiera el

9 Pérez Tamayo, R., De la magia primitiva a la medicina moderna, cit., nota 3, Ciencia para Todos, n. 154. parte tercera, p. 169.

10 Vögele, J., "Zur Entwicklung der Gesundheitsverhältnisse im 19. und 20. Jahrhundert", Geschichte der Therorie und Ethik der Medizin, Hrsg. Stefan Schulz, Klaus Steigleder, Heine Fangerau und Nobert W. Paul. Suhrkamp Frankfurt, 2006, pp. 165-183.

11 Idem.

12 Price, D., "Ciencia y tecnología: distinciones e interrelaciones", en Barnes, Barry et al. (ed.), Estudios sobre sociología de la ciencia, Madrid, Alianza Universidad, 1980, pp. 163-178. 
médico, o el equipo de médicos, cuenta con toda la información pertinente sobre un caso clínico, de tal manera que pueda decirse que el conocimiento médico es determinante en la toma de decisiones. Este inconveniente vale para cualquiera de las especialidades clínicas, especialmente para los grandes campos de la medicina interna o medicina general. ${ }^{13}$ La segunda es que si no se pueden presentar los motivos, fundados en "todo" el conocimiento teórico científico disponible y relevante, por los cuales se tomaron las decisiones en la práctica médica, entonces la primacía en la toma de decisiones la tiene el aspecto ético-normativio. ${ }^{14}$

La historia de la medicina constata que el flujo de conocimiento biomédico (o el conocimiento proveniente de cualquier otra ciencia natural) no constituye ninguna garantía para resolver problemas de la práctica médica o, por lo menos, para hacerla más fácil. ${ }^{15}$ Sin embargo, no debe cejar la exigencia, tanto por parte de la calidad y seguridad de la práctica médica, como también desde el punto de vista ético, de establecerse relaciones causales entre conocimiento y práctica médica con el fin de "justificar" y "fundamentar" las decisiones en cada uno de los casos clínicos. ${ }^{16}$ Por esta razón, se presenta en este punto el dilema del exceso de conocimiento como rasgo problemático de la relación entre conocimiento y praxis médica, que puede ser descrito como un defecto sistémico entre el plano teórico y el plano práctico. ${ }^{17}$

13 Paul, W. N., "Medizintheorie", Geschichte der Therorie und Ethik der Medizin, Hrsg. Stefan Schulz, Klaus Steigleder, Heine Fangerau und Nobert W. Paul. Suhrkamp Frankfurt, 2006, pp. 59-77.

14 Véase Canguilhem, G., Lo normal y lo patológico, México, Siglo XXI, 1970, segunda parte, c. IV, p. 137.

15 Sigerist, H., Civilización y enfermedad, México, Fondo de Cultura Económica, 1946, c. V, p. 135.

16 Paul, W. N., "Diagnose und Prognose", Geschichte der Therorie und Ethik der Medizin. Hrsg. Stefan Schulz, Klaus Steigleder, Heine Fangerau und Nobert W. Paul. Suhrkamp Frankfurt, 2006, pp. 143-155.

17 Idem. 
Esta relación entre la investigación biomédica y el quehacer clínico la presenta Norbert Paul ${ }^{18}$ como si se tratara de la relación de dos mundos independientes y sólo algunas veces complementarios. Paul agrega, además de la razón mencionada, las presiones del médico en cada situación clínica al pie de la cama del enfermo, las cuales imposibilitan una relación completa y directa de la praxis médica a partir del conocimiento médico y de las teorías que lo fundamentan.

Una vez llegado a este punto, se plantea nuevamente la pregunta sobre la relación entre conocer y obrar en la medicina de la siguiente manera: ¿cómo puede ser empleado el conocimiento biomédico, por ejemplo, como instancia de control y criterio determinante para la práctica clínica en la medicina moderna, cuando ambos están sólo superficialmente conectados?

Este fue el típico planteamiento de la teoría de la ciencia médica de inspiración analítica. ${ }^{19}$ En esta teoría se asumió que la explicación exacta de las relaciones entre teoría y práctica clínica constituía el problema central de la medicina científica y que, para resolverlo, es necesaria una reconstrucción teórica de la forma como se proyecta el conocimiento biomédico en la práctica médica. ${ }^{20}$ La repercusión de esta postura se aprecia en los principios propuestos para fundamentar la ciencia médica, a saber: exactitud analítica y causalidad. Ambos serían constitutivos de la cientificidad de la medicina, y ambos impactarian tanto en el campo de la teoría biomédica como en la práctica clínica.

El primer principio, el de la exactitud analítica, consiste en que sólo el conocimiento científico comprobado, obtenido

18 Paul, W. N., "Der Hiatus theoreticus der naturwissenschaftlichen Medizin. Vom schwierigen Umgang mit wissen in der Humanmedizin der Moderne”, Anatomien medizinischen Wissen, editado por Cornelius Borck. Frankfurt am Main, 1996, pp. 171-200.

19 Esta postura fue defendida por Karl Eduard Rothschuld y Fritz Hartmann. Rothschuh, K., E. K., Was ist Krankheit? Erscheinung, Erklräung, Sinngebung. WBG. Darmstadt, 1975.

20 Idem. 
con métodos y que utilice términos bien definidos, será considerado condición suficiente para fundamentar la práctica médica. El segundo principio, el de causalidad, está dirigido ante todo a regular la práctica médica a través del vínculo con el saber. De esta forma, los casos de la práctica médica que pueden recibir una fundamentación científica serán aquellos que estén adecuadamente descritos y clasificados, o sea, referidos a conceptos de la medicina científica. La visión analítica de la teoría médica piensa que sólo de esta manera quedarán científicamente diagnosticadas las molestias de un paciente, o sea, sólo de esta manera quedarán "justificadas" y "fundamentadas" las decisiones del médi$\cos ^{21}$

Por el lado de la teoría médica clásica, ${ }^{22}$ también se considera que debe ser posible validar la práctica médica sometiéndola tanto el principio de la exactitud analítica como al principio de la causalidad. Su planteamiento del problema sería el siguiente: ¿pueden ser presentadas, y de qué manera, los principios generales que rigen necesaria y suficientemente una práctica clínica? ${ }^{23}$

Lo interesante en la propuesta de la teoría médica clásica reside en que su motivación, para el planteamiento del problema, fue la convicción de que es posible imaginar la presencia de problemas éticos en aquellas circunstancias en las que hay un abismo entre teoría y praxis, o sea, donde las decisiones clínicas son tomadas sobre la base de conocimientos vagos y, por tanto, asumiendo un gran riesgo.

Tanto el planteamiento analítico, como el de la teoría clásica, fueron puestos en duda por el antipositivismo, desarrollado principalmente hacia mitad de la década de los se-

21 Paul, W. N., "Der Hiatus theoreticus der naturwissenschaftlichen Medizin. Vom schwierigen Umgang mit wissen in der Humanmedizin der Moderne", Anatomien medizinischen Wissen. Editado por Cornelius Borck, Frankfurt am Main, 1996, p. 180.

22 Hartmann, F., "Krankheitsgeschichte und Krankengeschichte", Sitzungsberichte der Gesellschaft zur Beförderung der gesamten Naturwissenschaften zu Marburg, Huber-Verlag. Marburg, 1966, pp. 17-32.

23 Idem. 
senta del siglo XX. ${ }^{24}$ Quien hizo posible este cambio en la producción científica bajo una nueva luz fue Ludwik Fleck con su tratado "Origen y desarrollo del hecho científico" 25 de 1935. También el físico Thomas Kuhn ${ }^{26}$ permitió entender de forma diferente el desarrollo de la teoría médica con su obra La estructura de las revoluciones científicas. Esto es, fueron sobre todo los trabajos inspirados en la historia de la ciencia los que permitieron una nueva mirada en los problemas de la teoría médica.

De esta manera, el antipositivismo creyó necesario describir la práctica científica en su realización misma y no más en el plano teórico, pues consideraba que las teorias anteriores se habian ocupado con un tipo ideal e inexistente de ciencia, para obtener de ahí un criterio de "cientificidad" y luego aplicárselo a toda disciplina académica. Los estudios histórico-científicos cambiaron el acento dentro de las investigaciones sobre la ciencia, reclamando de manera insistente que se orientara según los resultados empíricos de la investigación científica. Esto implicaba también tomar distancia del racionalismo crítico de Karl Popper. ${ }^{27}$

Gracias a este nuevo enfoque, actualmente se caracteriza la investigación científica médica de la siguiente manera:

1. Realización de la práctica médica como práctica social.

2. Consideración de la práctica social para el criterio de conocimiento científico.

3. Elaboración de tecnologías para la práctica científica.

4. Importancia del laboratorio en el proceso de adquisición del conocimiento.

24 Fleck, L., Erfahrung und Tatsache, Frankfurt, Gesammelte Aufsätze. Suhrkamp, 1989, pp. 76-130.

25 Fleck, L., "Entstehung und Entwicklung einer wissenschaftlichen Tatsache. Einführung”, in die Lehre vom Denkstil und Denkkollektiv. Suhrkamp, Frankfurt, 1975 (reimpresión), pp. 17-30.

26 Kuhn, Th., La estructura de las revoluciones científicas, México, Fondo de Cultura Económica, 1980.

27 Esta temática dio lugar a una larga y fructífera discusión sobre el método científico, entre, por un lado, Popper, y, por otro, Paul Feyerabend. Popper, K., Logik der Forschung, Berlín, Mohr Siebeck, 2001. Feyerabend, P., Wider den Methodenzwang, Frankfurt a. m., Suhrkamp, 1999. 
Con esto nuevamente se pretende que nunca más aparezca la teoría y la práctica médica como dos esferas separadas e independientes, sino, por el contrario, que ambas sean entendidas como inseparables dentro de la estricta producción de los hechos científicos. Pero en este caso, se cree que esto es posible porque, dentro de esta nueva visión del proceso de creación de conocimiento, las ciencias naturales, la medicina y sociedad se implican mutuamente. ${ }^{28}$ Cada ámbito será entendido como producto y productor de las condiciones tanto científicas, como médicas y culturales, responsables de la normatividad de la función científica y de la determinación de conceptos médicos que operan en la práctica.

Bajo este nuevo repertorio histórico-cultural de los modelos explicativos científicos serán interpretados nuevamente los conceptos normativos salud/enfermedad, claves de la teoría y práctica médica. Esta nueva interpretación será a su vez relevante para una nueva lectura de la ciencia médica toda.

\section{NUEVA LECTURA DE LA CIENCIA MÉDICA DESDE LOS CONCEPTOS NORMATIVOS SALUD/ENFERMEDAD}

La reflexión sobre los conceptos normativos salud/enfermedad representa para la teoría médica un aspecto tan fundamental como la reflexión sobre la relación entre conocimiento y praxis médica. ${ }^{29}$ Incluso los historiadores y teóricos de la medicina, pertenecientes a la corriente analítica y clásica, se ocuparon de estudiar el significado de estos conceptos fundamentales. ${ }^{30}$ Curiosamente, fue al interior de las investigaciones científicas donde cobró importancia los temas de la historia de la ciencia y la teoría e historia de

28 Sigerist, H., Civilización y enfermedad, México, Fondo de Cultura Económica, 1946, pp. 15-57.

29 Canguilhem, G., Lo normal y lo patológico, México, Siglo XXI, 1971, primera parte, pp. 17-73.

30 Rothschuh, K. E., Prinzipien der Medizin. Ein Wegweiser durch die Medizin. Frankfurt a. m., Urban u. Fischer, 1982, p. 189. 
la medicina, no obstante que allí mismo fue donde en un principio se pretendió restarles importancia. La razón que impulsó este interés fue precisamente la imposibilidad de alcanzar el ideal científico de fundamentar las relaciones causales en la medicina mediante condiciones necesarias y suficientes; fundamento que serviría, a su vez, a la toma de decisiones en la praxis médica.

En la medicina representa el concepto de salud una categoría teleológica, en la que convergen todas las prácticas médicas: el fin es sanar. En contraparte, el concepto de enfermedad representa una categoría de carácter "legitimatorio", desde la cual se justifica y construye la intervención médica. ${ }^{31}$ No se pierde de vista que entre más variados sean los criterios sobre la medicina moderna, más proliferará la inseguridad sobre la finalidad de la práctica médica, así como los riesgos de su intervención. Considerando estos dos elementos, resulta evidente que ni la ciencia ni la ética médica pueden continuar negando su interdependencia, de la cual se tendrá un mejor entendimiento mediante el análisis de los conceptos fundamentales salud/enfermedad. Por eso, es necesario insistir, una vez más, en la relevancia del punto de vista de la investigación científica moderna dentro de su contexto ético-histórico-cultural para la compresión de estos dos conceptos. ${ }^{32}$

\section{LA TEORÍA DE LA MEDICINA FRENTE A LA APERTURA INTERPRETATIVA DE SALUD Y ENFERMEDAD}

La interpretación de síntomas patológicos se encuentra en un proceso permanente e inacabable de redefinición. La pregunta sobre cómo se ha entendido salud y enfermedad en la ciencia moderna y qué consecuencias ha tenido esto

31 Paul, W. N., "Medizintheorie", en Geschichte der Therorie und Ethik der Medizin. Hrsg. Stefan Schulz, Klaus Steigleder, Heine Fangerau und Nobert W. Paul. Suhrkamp Frankfurt, 2006, pp. 131-143.

32 Sigerist, H., Civilización y enfermedad, México, Fondo de Cultura Económica, 1946, introducción, pp. 9 y ss. 
para la práctica médica, no es posible de responder teóricamente sino a partir de la observación de casos concretos. ${ }^{33}$ Con esto nuevamente se justifica la exigencia de que la teoría médica se informe acerca de la historia y de la moral. Esta exigencia también es una consecuencia de la variación e inestabilidad de los conceptos fundamentales salud/enfermedad dentro de diferentes contextos sociales, culturales, locales e históricos. Por eso, puede decirse que los conceptos salud y enfermedad están en cierta manera siempre abiertos a la interpretación.

Esta circunstancia representa un problema para planteamientos tradicionales de la medicina y teoría de la ciencia de corte clásico y analítico, que confiaban en la amplitud de los modelos explicativos de la biomedicina. ${ }^{34} \mathrm{El}$ esclarecimiento de estos conceptos fundamentales exigía, según ellos, la descripción exacta y completa tanto de un organismo humano sano como de otro enfermo. Pero una teoría semejante del organismo humano es imposible en razón de la apertura del organismo a las influencias variables del entorno y a su propio comportamiento frente al mismo. ${ }^{35}$

También puede esgrimirse que una fundamentación teórica completa de los conceptos salud/enfermedad es muy difícil simplemente debido al factor multicausal de las enfermedades, incluso pese a que hayan aparecido algunos planteamiento aislados de la teoría clásica de la medicina precisamente con esta pretensión. ${ }^{36}$ No se niega que estos planteamientos colaboraron en su momento al entendimiento de las distintas funciones de la medicina, ${ }^{37}$ pero la exigencia misma de fundamentar la medicina o, por lo me-

33 Perez Tamayo, R., Enfermedades viejas y enfermedades nuevas, México, Siglo XXI, 1985, capítulo I, pp. 11 a 61.

34 Fleck, L., Erfahrung und Tatsache. Gesammelte Aufsätze, Frankfurt, Suhrkamp, 1989, pp. 66-77.

35 Sigerist, H., Civilización y enfermedad, México, Fondo de Cultura Económica, 1946, c. I y II.

36 Pérez Tamayo, R., Enfermedades viejas y enfermedades nuevas, México, Siglo XXI, 1985, capítulo I, pp. 61-98.

37 Porter, R. y Vigarello, G., "Cuerpo, salud, enfermedad”, Historia del cuerpo, vol. I: Del Renacimiento a la Ilustración, Madrid, Taurus, 2005, pp. 323-358. 
nos sus conceptos cardinales (salud/enfermedad), no fue ni ha podido ser lograda.

En razón de esos problemas teóricos fundamentales, se han empleado en la ciencia médica los conceptos salud/enfermedad siguiendo aquellas consideraciones pragmáticas que se han distinguido por su gran funcionalidad a la hora de resolver problemas clínicos. Frente a este panorama, la teoría de la medicina se ha fijado dos tareas: por un lado, se ocupa ahora con determinadas entidades cognoscitivas con el fin de indagar su valor explicativo; 38 por otro lado, se ha impuesto la tarea de analizar los conceptos salud/enfermedad dentro del ámbito de competencia de medicina humana moderna. 39

Como se ha dicho, los conceptos salud/enfermedad se encuentran en estado dinámico, o sea, son conceptos "abiertos”, influenciados tanto por nuevos conocimientos científicos, por parte de las biociencias, como por conocimientos provenientes de las ciencias sociales y humanidades. Adicionalmente, existe un intercambio difícil de aprehender entre las representaciones populares sobre enfermedad y aquellas forjadas en las ciencias naturales. ${ }^{40}$ Este bagaje de información influye en la interpretación de salud y enfermedad en todas las ciencias, sea que se piense en la medicina o en la psicología o en la psiquiatría, etcétera, porque en todos los casos se trata de saber cuándo puede ser llamado un hombre sano o enfermo. ${ }^{41}$

La consecuencia es que los planteamientos modernos provenientes de las ciencias médicas, como la concepción de la patología biomédica sobre la enfermedad, son uno entre mu-

38 Este es el planteamiento acostumbrado que encuentra repercusión en el ámbito patológico. Véase Paul, W. N., "Medizintheorie”, cit., nota 31, p. 61.

39 Idem.

40 Handlin, O., "La ambivalencia en la reacción popular ante la ciencia"; y también: Rosenberg, E., "Las teorías científicas y el pensamiento social", ambos en Estudios sobre sociología de la ciencia, editado por Barnes, Barry et al., Madrid, Alianza Universidad, 1980, pp. 247-262, y 284-299, respectivamente.

41 Rothschuh, K., Physiologie im Werden, Frankfurt a. m., Urban \& Fischer Verlag, 1998, pp. 139 y ss. 
chos posibles, además que nunca llegarán a ser estos planteamientos los que dominen sobre el sentido mundano de enfermedad, 42 por lo menos no a la manera como sí lo hace en la medicina clínica. Esto también ya fue aceptado en la perspectiva constructivista de la teoría médica antipositiva, ${ }^{43}$ porque se admite que sería insostenible interpretar los conceptos salud/enfermedad simplemente como una construcción social. Esto representaría ignorar el carácter material de la enfermedad, así como su dependencia respecto del organismo, de la conducta, y de sus órganos, tejidos, células, moléculas, etcétera. Por tanto, una interpretación puramente social de los conceptos salud/enfermedad no puede manejar las distintas interpretaciones de estos conceptos en la mayor parte de los discursos. Su intento de definición es el siguiente:

Salud es la construcción sin molestia del mundo subjetivo, a partir de lo que el entorno ofrece de útil y dañino. Esto corresponde a la capacidad creativa del organismo vivo. Por el contrario, la enfermedad aparece cuando es destruido el equilibrio entre la capacidad subjetiva de creatividad y la oferta objetiva del entorno,...44

\section{RELACIÓN ENTRE TEORÍA Y ÉTICA MÉDICA CON BASE EN LOS CONCEPTOS SALUD/ENFERMEDAD}

Los conceptos salud/enfermedad constituyen un problema que coloca a la teoría de la medicina en la necesidad de conectar por lo menos dos sistemas teóricos "independientes": práctica de la ciencia médica y ética. La razón reside en que los conceptos salud/enfermedad subrogan los valo-

42 Pérez Tamayo, R., Enfermedades viejas y enfermedades nuevas, cit., nota 33, capítulo I, pp. 11-61.

43 Fleck, L., Entstehung und Entwicklung einer wissenschaftlichen Tatsache. Einführung in die Lehre vom Denkstil und Denkkollektiv, Frankfurt, Suhrkamp, 1975, pp. 67-130.

44 Idem. 
res ético-sociales al orden interno de la corporeidad, porque el sentido de los términos salud/enfermedad se traduce en comportamiento social y en exigencias ético-sociales respecto del cuerpo. ${ }^{45}$ Los términos salud/enfermedad dejan ver la frontera donde se transforma la ciencia en orden social, o sea, en normatividad del cuerpo. De esta manera, puede afirmarse que no existe una experiencia corporal natural o científica, que suponga una percepción "directa" de la salud y enfermedad sin influencia de la vida cultural.

En la medida en que la ciencia ha definido la relación del hombre con la naturaleza, se ha ampliado la competencia de la ciencia médica dentro de la sociedad, con el fin de que esta última devenga más y más territorio exclusivo de su interpretación y dominio. Interpretación y dominio ejercido a través principalmente de los conceptos salud/enfermedad. ${ }^{46}$ De aquí resulta un control de la vida sobre la base de la medicación, justificada en la ciencia y tecnológica médica. ${ }^{47}$ La consecuencia de esto es la disolución de la diferencia entre público y privado, y el control absoluto de la ciencia médica sobre sus pacientes.

Se debe a estudios sobre la práctica médica, como los de Georges Canguilhem ${ }^{48}$ o Henry Sigerist, ${ }^{49}$ el que hoy día sea visible la manera en que operan los conceptos normativos salud/enfermedad dentro de la práctica médica y la sociedad. Estos autores muestran la invasión de la ciencia médica en la sociedad, la forma en que amplía su tarea y objeto de estudio al límite de definir lo que es el hombre como hombre; porque si bien la intervención médica se justifica siempre y cuando exista una enfermedad, la fragilidad del

45 Sigerist, E. H., Civilización y enfermedad, México, Fondo de Cultura Económica, 1946, cap. IV, pp. 106-135.

46 Marcuse, H., "La racionalidad tecnológica y la lógica de la dominación”, Estudios sobre sociología de la ciencia, editado por Barnes, Barry et al., Madrid, Alianza Universidad, 1980, pp. 323-344.

47 Foucault, M., Los anormales. Curso en el Collége de France 1974-1975, México, Fondo de Cultura Económica, 2001, pp. 187-215.

48 Canguilhem, G., Lo normal y lo patológico, México, Siglo XXI, 1970.

49 Sigerist, E. H., Civilización y enfermedad, México, Fondo de Cultura Económica, 1946. 
organismo precisa de la periódica inspección médica. Usos $\mathrm{y}$ abusos de la sociedad se encuentran literalmente bajo análisis microscópico. La mejor manera de saber que es debido o reprobable consiste en consultar al médico. Su inapelable sabiduría científica deshecha las recomendaciones y sugerencias; o bien se ajusta la vida a sus prescripciones, o se acepta irresponsablemente el riesgo de perderla; y quien a sabiendas de lo que debe hacer, desoye los preceptos de la consulta médica, puede ser objeto de persecución judicial. 50

Ahora bien; una vez diagnosticada la enfermedad, la persona no es más autónoma y se convierte en "paciente".51 Esto quiere decir que a partir de ese momento se considera a los médicos representantes de los intereses del hospitalizado. También a partir de ese momento, se espera que el médico, con la aplicación de sus conocimientos y experiencia en la práctica médica, cure los malestares que sus pacientes no pueden por sí mismos superar, y repare la "autonomia" del paciente $\mathrm{y}$, por tanto, la reconstrucción de su situación social toda. Huelga decir que de este momento en adelante la "autonomía" y situación social del paciente permanecerán bajo la constante inspección médica, misma que no tiene por qué verificarse en el consultorio médico, ya que cualquier indicación habitual de su entorno, como "no fumar", "no beber", "no correr", "no pasar", estará todo amparado en una advertencia médica. 52

De esta forma es como la ciencia y tecnología rebasan su primitivo ámbito "mecánico" de competencia, dilatándose, por ejemplo, al orden judicial con un nuevo concepto de

50 Foucaullt, M., Los anormales. Curso en el Collége de France 1974-1975, cit., nota 47, pp. 39-61.

51 Krones, T. y Richter, G., "Die Artz-Patient-Beziehung”, Geschichte der Therorie und Ethik der Medizin. Hrsg. Stefan Schulz, Klaus Steigleder, Heine Fangerau und Nobert W. Paul. Suhrkamp Frankfurt, 2006, pp. 94-117.

52 Marcuse, H., "La racionalidad tecnológica y la lógica de la dominación", Estudios sobre sociología de la ciencia, editado por Barnes, Barry et al., Madrid, Alianza Universidad, 1980, pp. 323-344. 
justicia imparcial:53 "justo es exigir a cualquiera lo que está médicamente fundamentado”.

Esta es la razón del requerimiento de hacer analizar la relación entre conocimiento y práctica médica dentro de su contexto social, acentuando la reflexión ética con el fin de lograr una explicitación y evaluación de los criterios normativos implicados en esa actividad. Y como los conceptos salud/enfermedad determinan el lugar y el alcance normativo de la práctica médica en una sociedad, será de ellos principalmente que se ocupe la teoría de la medicina para dilucidar la influencia de la ciencia médica en el hombre y sociedad. 54

Es claro, pese a lo dicho, que persistirá la tentación de aclarar los conceptos salud/enfermedad a partir únicamente de la armonía entre conocimiento teórico y acción o práctica médica, bajo la creencia de que de esta manera la acción de los médicos se orienta simplemente a la reparación de la "máquina humana", sin otras secuelas para el paciente y la sociedad. 55 Pero esto, como se vio más arriba, no es posible; y la influencia de la sociedad en los "sistemas científicos" continuará transformando las cuestiones de ciencia médica en asuntos de ética social.

53 Foucault, M., Los anormales. Curso en el Collége de France 1974-1975, cit., nota 47, pp. 131-157.

54 Habermas, J., "Ciencia y tecnología como ideología", Estudios sobre sociología de la ciencia, editado por Barnes, Barry et al., Madrid, Alianza Universidad, 1980, pp. 344-365.

55 Arasse, D., "La carne, la gracia, lo sublime", Historia del cuerpo, vol. I: Del Renacimiento a la Ilustración, Madrid, Taurus, 2005, pp. 416-427. 